\title{
Trans fatty acids and coronary heart disease
}

\author{
Food labels should list these as well as cholesterol and saturated fat
}

A recent systematic review by Mozaffarian and colleagues in the New England Journal of Medicine advocated that people should reduce or stop their dietary intake of trans fatty acids to minimise the related risk of coronary heart disease. ${ }^{1}$ Trans fatty acids-unsaturated fatty acids with at least one double bond in the trans molecular configuration-are produced by partial hydrogenation of vegetable oils. This is a process that converts vegetable oils into semisolid fats which have no known nutritional value but are widely used in margarines, in commercial cooking, and in manufactured foods. ${ }^{2}$

Reliable evidence for the effects of trans fatty acids and other types of fat on blood lipids comes from the "metabolic ward" studies (in which participants are admitted to wards specialising in metabolic studies). Typically, participants in these studies consumed isocaloric diets under controlled conditions that exchanged the amount and type of fat for complex carbohydrate and the effects on participants' blood lipids were then measured. These trials showed that increasing intakes of saturated fat, trans fat (trans fatty acids), and dietary cholesterol raises total cholesterol and low density lipoprotein (LDL) cholesterol concentrations; polyunsaturated fats lower LDL concentrations; monounsaturated fats have a neutral effect on LDL; and eliminating trans fatty acids from the diet reduced total cholesterol by $0.1 \mathrm{mmol} / \mathrm{l}^{2-4}$ Recent evidence on the relation between dietary trans fatty acids and blood lipids has shown that trans fatty acids increase LDL and reduce high density lipoprotein (HDL) cholesterol concentrations. Compared with equivalent changes in intake of saturated fats, intake of trans fatty acids has a more adverse effect on the ratio of total cholesterol to HDL cholesterol concentrations. ${ }^{12}$

The review by Mozaffarian and colleagues included a meta-analysis of prospective studies of trans fatty acids and risk of coronary heart disease. A $2 \%$ increase in the energy intake from trans fatty acids was associated with a $23 \%$ increase in the incidence of coronary heart disease (pooled relative risk 1.23; 95\% confidence interval 1.11 to 1.37$){ }^{1}$ The authors noted that the adverse effects of trans fatty acids were seen even when intake was really low, only $3 \%$ of total daily energy intake (20-60 calories), about 2-7 $\mathrm{g}$ for a person consuming 2000 calories $(8.37 \mathrm{~mJ}$ ) per day.

Legislation introduced in Denmark in 2004 mandated that all oils and fats used in locally made or imported foods must contain less than $2 \%$ industrially produced trans fatty acids. This virtually eliminated trans fatty acids and had no effect on quality, cost, or availability of foods. In January 2006 the US Food and Drug Administration mandated that all food manufacturers provide the content of trans fatty acids and cho-
Average percentage of saturated fatty acid and trans fatty acid, by weight, in selected foods in the UK diet ${ }^{5}$

\begin{tabular}{lccc} 
Food & Saturated fats & Trans fats & Both fats \\
\hline Spreads: & & & \\
\hline Butter & 52 & 3 & 55 \\
\hline Hard margarine & 35 & 12 & 47 \\
\hline Fat spreads & 11 & 3 & 14 \\
\hline Biscuits: & & & \\
\hline Chocolate caramel biscuit bar & 12 & 5 & 17 \\
\hline Chocolate biscuit & 12 & 2 & 14 \\
\hline Digestive plain & 9 & 1 & 10 \\
\hline Oat and wheat bar with fruit filling & 2 & trace & 2 \\
\hline Savoury snacks: & & & \\
\hline Crisps & 14 & $?$ & 14 \\
\hline Cream crackers & 5 & 1 & 6 \\
\hline Mixed nuts & 8 & 0 & 8 \\
\hline
\end{tabular}

lesterol in addition to saturated fat on nutrition labels for all manufactured foods

The UK Food Standards Agency is currently pressing for revision of the European directive that governs the content and format of nutrition labels on foods marketed in the United Kingdom and other European countries, so that these fats are labelled. The trans fatty acid content of commonly used, randomly selected foods consumed in the UK up to 2002 varied substantially between one type and another (table ${ }^{5}$ ). Some margarines may now have lower concentrations of trans fatty acids, but consumers need to know which ones to choose. Mandatory addition of the content of saturated fat and trans fatty acids to nutrition labels would enable consumers to make healthier food choices that could lower LDL concentrations and reduce the risk of coronary heart disease and other vascular events.

\section{Robert Clarke honorary consultant in public health} medicine

(robert.clarke@ctsu.ox.ac.uk)

Sarah Lewington senior research fellow

Clinical Trial Service Unit and Epidemiological Studies Unit, Richard Doll Building, University of Oxford, Oxford OX3 7LF

Competing interests: None declared.

1 Mozaffarian D, Katan ME, Ascherio A, Stampfer MJ, Willett WC. Tran fatty acids and cardiovascular disease. N Engl J Med 2006;354:1601-13.

2 Mensink RP, Zock PL, Kester ADM, Katan MB. Effects of dietary fatty acids and carbohydrates on the ratio of serum total to HDL cholesterol and on serum lipids and apolipoproteins: a meta-analysis of 60 controland on serum lipids and apolipoproteins:

3 Clarke R, Frost C, Collins R, Appleby P, Peto R. Dietary lipids and blood cholesterol: a quantitative meta-analysis of the metabolic ward studies. BMJ 1997;314:112-7.

4 Clarke R, Frost C. Dietary fat, blood lipids and coronary heart disease risk. In: Wilson T, Temple N, eds. Nutritional health:strategies for disease prevention. Totowa, NJ: Humana, 2001.

5 Food Standards Agency. McCance and Widdowson's The composition of foods. 6th ed. Cambridge: Royal Society of Chemistry, 2002. 